\title{
Observational study of newborn infant parasympathetic evaluation as a comfort system in awake patients admitted to a pediatric intensive care unit
}

\author{
Juan Valencia-Ramos ${ }^{1}$ (D) $\cdot$ Juan Arnaez $^{2,3} \cdot$ Sara Calvo $^{4} \cdot$ Fernando Gomez $^{1} \cdot$ Isabel Del Blanco $^{1}$
}

Received: 21 August 2018 / Accepted: 25 January 2019 / Published online: 5 February 2019

C) Springer Nature B.V. 2019

\begin{abstract}
To compare the newborn infant parasympathetic evaluation system (NIPE) scores with a validated clinical scale using two different nebulizers in children with bronchiolitis admitted to a PICU. Comfort was evaluated using the COMFORT-behavior scale (CBS) before (T1), during (T2) and after (T3) each nebulization. In order to compare NIPE and CBS values during the whole T1 to T3 period, the variable Dif-CBS was defined as the difference between maximal and minimal CBS scores, and Dif-NIPE as the difference between 75th and 25th percentile NIPE values. Analyses were carried out, firstly for the total of nebulizations and secondly comparing two different nebulization systems: a jet nebulizer (JN) and a nebulizer integrated in high flow nasal cannulas (NHF). 84 nebulizations were recorded on 14 patients with a median [25th-75th percentile] age of 6 months (3.1-9.5). A Dif-CBS of 4 points (2-7), as well as changes in CBS scores between T1 and T2, defined the nebulization as a discomfort stimulus. The NIPE system, represented as the Dif-NIPE, showed a median variation of 9 points (7-10), and was poorly correlated to Dif-CBS $\left[\mathrm{r}_{\mathrm{s}} 0.162(\mathrm{P}=0.142)\right]$. Discomfort during nebulization, assessed by CBS was greater with the JN system compared to NHF: $17(13-22)$ vs $13(9-15)(\mathrm{P}=0.001)$. NIPE monitoring detected no significant differences between both nebulization systems $(\mathrm{P}=0.706)$. NIPE monitoring showed a variation in comfort during nebulization in the patient with bronchiolitis, though correlation with CBS was poor. Further research is required before NIPE can be suggested as a comfort monitoring system for the awake infant.
\end{abstract}

Keywords Comfort · Newborn infant parasympathetic evaluation · COMFORT Behavior Scale · Nebulization · Bronchiolitis

\section{Introduction}

Bronchiolitis is an acute inflammatory injury of the bronchioles caused by a viral infection in infants [1]. It is the most frequent cause of lower respiratory tract infection in infants and the predominant reason for admission of children under 1 year of age $[2,3]$. Of these, $3-11 \%$ will require admission to a pediatric intensive care unit (PICU) [4]. Unfortunately,

Juan Valencia-Ramos

jvalenra@gmail.com

1 Pediatric Intensive Care Unit, Burgos University Hospital, Avenida Islas Baleares s/n, CP 09006 Burgos, Spain

2 Department of Neonatology, Burgos University Hospital, Burgos, Spain

3 Fundación NeNe, Madrid, Spain

4 Research Unit, Burgos University Hospital, Burgos, Spain specific therapies are not available and only a few symptomatic strategies are extensively used, including oxygen, hydration, and nasal suction $[5,6]$.

Nebulization with salbutamol, epinephrine, or 3\% hypertonic saline is the most commonly used therapy in clinical practice, despite its doubtful efficacy, with the aim of reducing clinical severity $[7,8]$. However, nebulizations are not harmless because they are usually administered through a jet nebulizer that generates discomfort [9], due to its noise, with frequent awakening and struggling to remove it. This discomfort might be especially counterproductive in critically ill patients when trying to achieve desired stability [10].

The gold standard to assess patient comfort is self-reporting, based on pain scales. However, self-reporting is unlikely in pre-verbal children, as well as in infants with altered consciousness [11]. Regardless of which tool is used to assess pain, perception of pain in pediatrics is complex, and entails physiological, behavioral, and developmental factors [12, 
13], including the limitation of the experience of the examiner. In these instances, a cooperation-independent monitor for pain would be highly helpful [14]. The Newborn Infant Parasympathetic Evaluation (NIPE, MDMS, Loos, France) is a valid non-invasive system in children up to 2 years of age based on the analysis of the respiratory fluctuations of heart rate that mainly reflect variability in the parasympathetic tone [15]. The NIPE monitor records the ECG signal continuously, enabling a quantitative assessment of the respiratory variability of heart rate, which decreases during nociceptive stimulation [16]. To date, only a few studies have focused on this technology in children, the majority of them in the area of anesthesia [17-19].

The aim of this study was (1) to evaluate whether the NIPE system could be a useful tool for the continuous monitoring of discomfort and pain, compared to the clinical COMFORT Behavior Scale when nebulizing children with bronchiolitis admitted to a PICU; and (2) to determine whether NIPE values vary when two different nebulization systems are used.

\section{Methods}

\subsection{Participants}

Infants with bronchiolitis and treatment with High Flow Nasal Cannulas (HFNC) and aerosol therapy were enrolled in this prospective observational study in the PICU of a tertiary university hospital between January 2017 and May 2017. The use policy for nebulizations in children with bronchiolitis in our PICU is dependent on the physician responsible for the patient, an initial test generally being performed and prescribing nebulizations if the clinical response is suitable. Children more than 24 months old with arrhythmia or cardiac malformation were excluded from the study. This study respected the Helsinki Declaration and was approved by the local Institutional Review Board (Comité Ético de Investigación Clínica del Área de Salud Burgos y Soria, Ref: CEIC-1558). Informed consent was requested from parents.

\subsection{Study design}

After parental consent was obtained, subjects were randomized by a computer-generated random number list to begin the nebulization with a jet nebulizer (JN) or a nebulizer integrated in the HFNC system (NHF), alternating the nebulization device.

The NIPE monitor (NIPE, MDMS, Loos, France) was connected to the electrocardiogram and data recording enabled until nebulizations ended during admission. The algorithm used for NIPE computation has been previously described $[15,18]$. It expresses a numerical value between
0 and 100; high values correspond to high parasympathetic activity reflecting the wellbeing of the patient. The NIPE calculates two indexes: averaged value (NIPEm) for chronic or prolonged pain and instantaneous reading (NIPEi) for acute pain. We used the NIPEi to evaluate this procedure.

Clinical comfort was assessed using the COMFORT Behavior Scale (CBS) by nurses who had received specific training in this area, at 3 different times: $\mathrm{T} 1=5 \mathrm{~min}$ before, $\mathrm{T} 2=$ during, and $\mathrm{T} 3=5 \mathrm{~min}$ after the nebulization. Each CBS item is scored from 1 to 5 , and the final score range is the sum of the six behavioral items. This made 6 the lowest possible score (no pain) and 30 the highest (the greatest pain) [19].

\subsection{Measurement and analysis}

Our working hypothesis was that NIPE would detect changes in the level of discomfort or pain experienced during nebulization, and that its values would vary as clinical assessment scale scores had varied, depending on the type of nebulization system used [20].

The NIPE system stores the values obtained in each minute in its internal memory, as well as the quality of the signal at that time (Quality), such that ' 1 ' reflects an adequate recording signal and ' 0 ' reflects an inadequate signal. Once NIPE "Zero Quality” values had been removed from a total of 102 nebulizations on a total of 14 patients, the final sample size that was available for analysis amounted to 2833 NIPE values from 84 nebulizations performed on 14 patients. These measurements were evenly distributed amongst both nebulization systems: 1412 NIPE values from 40 nebulizations using NHF and 1421 NIPE values from 44 nebulizations using JN.

In order to analyze the changes in the comfort-discomfort, the 'difference' (Dif) variables were used. These variables (Dif-NIPE and Dif-CBS) were created so that NIPE and CBS comfort measurements could be compared during the whole nebulization period (T1 to T3). "Dif-CBS" was defined as the median of the differences between the maximum and minimum scores obtained when CBS was applied at each moment of the nebulization period (T1, T2 and T3). Dif-NIPE represented the difference between the 75th and 25th percentiles of the NIPE values during the same period as CBS (from T1 to T3).

Comfort levels were assessed using CBS and NIPE for all nebulizations as a whole and also for each of the two nebulization systems used (JN and NHF). A correlation study between the two comfort monitoring systems (clinical scale and NIPE monitoring) was made, and the two nebulization systems were compared (JN vs. NHF).

Other variables potentially associated with the comfortdiscomfort state were collected: date and time; food intake; administration of painkillers, whether the patient was being 
handheld by a parent or not; physiological variables (Heart Rate (HR), Respiration Rate (RR); and the administration of supplemental oxygen as fraction of inspired oxygen $\left(\mathrm{FiO}_{2}\right)$ ).

\subsection{Data analysis and statistics}

Absolute and relative frequencies were calculated for each qualitative variable, and differences based on nebulization system were identified using Chi square or Fisher's exact test. For quantitative variables, median [25th-75th percentile] were obtained according to nebulization system and time period. Spearman's correlation coefficient $\left(r_{s}\right)$ was used to measure correlation among the CBS (Dif-CBS) and NIPE values (DifNIPE), and nonparametric testing (Mann-Whitney's U test) was used to compare the two nebulization systems in each time period. ANOVA for repeated measures was used when CBS scores were compared during different moments within the same nebulization (T1, T2 and T3) for each of the two nebulization systems (NHC and JN). When Dif-CBS and Dif-NIPE were being compared, a "patient" variable was introduced in order to reflect the number of nebulizations administered to each individual patient. Values of $\mathrm{P}<0.05$ were considered to be statistically significant.

Statistical analyses were performed using statistics software (PASW Statistics 18.0, SPSS, Chicago, Illinois).

\section{Results}

A total of 84 nebulizations were recorded on 14 patients with bronchiolitis admitted to the PICU, (Table 1).

\subsection{CBS and NIPE monitoring}

CBS values increased during nebulization (T2) as compared to the period before (T1): median [25th-75th percentile] of 15 (11-19) Vs $13(9-15)(\mathrm{P}<0.001)$, with a difference of 4 points (2-7) when the whole period (from T1 to T3) was considered.

As regards to NIPE monitoring, Dif-NIPE was 9 points (7-10) during the whole T1 to T3 period. The correlation coefficient between Dif-NIPE and Dif-CBS was: $\mathrm{r}_{\mathrm{s}} 0.162$ $(\mathrm{P}=0.142)$ (Fig. 1).

Considering the 84 nebulizations, heart rate increased significantly from $133 \mathrm{bpm}(114 ; 144)$ before the nebulization $(\mathrm{T} 1)$ to $137(120.00 ; 151.00)$ during aerosol therapy (T2) $(\mathrm{P}=0.007)$. This variation between $\mathrm{T} 1$ and $\mathrm{T} 2$ for the CBS showed a correlation of $\mathrm{r}_{\mathrm{s}} 0.596(\mathrm{P}<0.001)$.

\subsection{Differences between the two nebulization systems (NHF and JN) using CBS and NIPE}

Of the 84 nebulizations, 40 were performed with the nebulization system integrated in the HFNC (NHF), and 44 with the conventional nebulizer (JN).
Table 1 Study population $(\mathrm{n}=14)$

\begin{tabular}{ll}
\hline Characteristics & Results \\
\hline Age (months) & $6(3.1-9.5)$ \\
Sex (male/female) & $7: 5$ \\
Weight (kg) & $7(6-9)$ \\
Height (cm) & $64(57-68)$ \\
Cephalic perimeter (cm) & $42(39-44)$ \\
Bronchiolitis scale ${ }^{\text {a }}$ & $8(7-10)$ \\
First episode of acute moderate to severe bronchiolitis & $9(64 \%)$ \\
Virus isolated & \\
Respiratory syncytial virus & $8(57 \%)$ \\
Rinovirus & $6(43 \%)$ \\
Metapneumovirus & $2(14 \%)$ \\
Coronavirus & $3(21 \%)$ \\
Parainfluenza 3 & $2(14 \%)$ \\
Adenovirus & $2(14 \%)$ \\
Enterovirus & $1(7 \%)$ \\
\hline
\end{tabular}

Quantitative variables are expressed in median (P25-P75), while categorical variables are expressed in $\mathrm{n}(\%)$

${ }^{a}$ Bronchiolitis Scale: San Juan de Dios Hospital-Bronchiolitis Scale $(0-5$ points $=$ mild, $6-10=$ moderate, $11-16=$ severe $)$

There were no significant differences at baseline between the groups (NHF and JN) when analyzing the time since last food intake, being held in the arms of relatives, time of day (day time-night time), use of analgesia, oxygen saturation, fraction of breathed oxygen, the breathing frequency, or heart rate before nebulization. Both nebulizers showed an increase in heart rate and breathing rate between $\mathrm{T} 1$ and $\mathrm{T} 2$, which was statistically significant in the case of JN $(\mathrm{P}=0.010)$ (Table 2).

During nebulization (T2) the CBS showed higher scores using JN compared with NHF: 17 (13-22) vs. 13 (9-15), respectively $(\mathrm{P}=0.001)$. JN CBS scores were higher $(\mathrm{P}=0.005)$ than those obtained using NHF in all moments of the nebulization period (T1, T2, T3).

The number of nebulizations with a CBS score $<17$ (53/84) was higher in the NHF group (33/53) than in the JN group $(20 / 53)(\mathrm{P}<0.001)$.

During the whole period (from T1 to T3), Dif-CBS was greater for NHF compared with JN: 3 (0-7) Vs 6 (2-9) $(\mathrm{P}=0.014)$.

No differences were found between the Dif-NIPE from T1 to T3 with NHF system and JN: 9 (7-10) vs 9 (7-10) $(\mathrm{P}=0.706)$, and correlation comparing the Dif-NIPE variables with Dif-CBS for each of the nebulizating systems was $r_{s} 0.272(P=0.089)$ and $r_{s} 0.116(P=0.452)$ for the NHF and JN systems, respectively. 
Fig. 1 The relationship between the difference in comfort levels measured using NIPE (DifNIPE) and the difference in comfort levels measured using CBS (Dif-CBS) from T1 to T3. Correlation between Dif-NIPE and Dif-CBS during T1-T3 period was: $\mathrm{r}_{\mathrm{s}} 0.162(\mathrm{P}=0.142)$

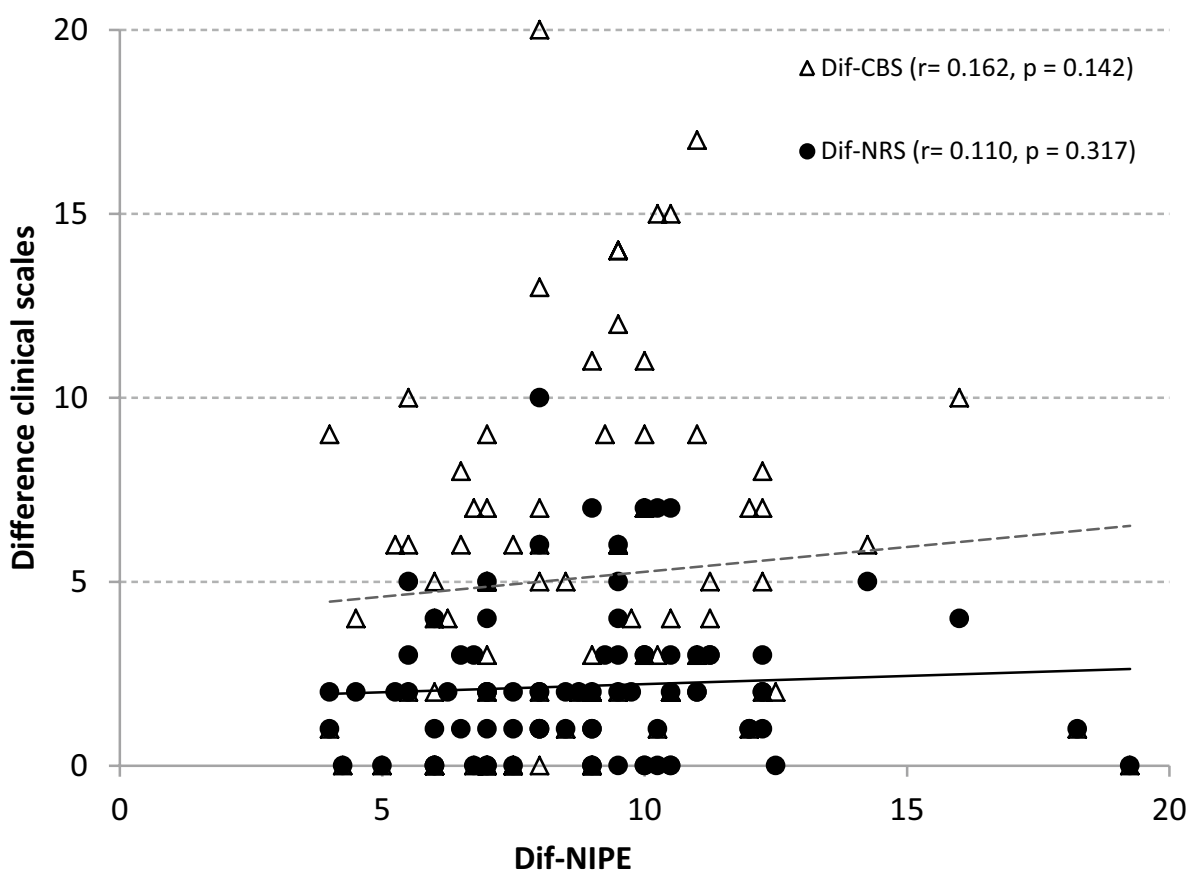

\section{Discussion}

Providing comfort in critically ill infants and children, and minimizing their anxiety, fear, and distress, are important parts of the daily activities in a PICU. However, in spite of its frequency, pain in this group of population is often underestimated and undertreated [21]. The aim of this study was to examine a tool that could shed some light in the challenging task of interpreting pain and discomfort in this patient group. The population chosen was infants with bronchiolitis admitted to PICU, with demographics similar to those described in previous studies [22]. The reason for choosing this disease, apart from its elevated incidence, was the frequent use in this group of treatments with potential discomfort, such as nebulizations [23]. In this paper, the results from a smaller previous study are corroborated, since it has been consistently found that nebulizations constitute a scource of discomfort for patients. The degree of this discomfort, as measured using clinical scales, is higher when using a JN system compared to a NHF system [20].

The Comfort Behavior Scale (CBS) was designed for use in pediatric populations with a similar age to the present study [24], and it is a valid and reliable tool for assessing comfort in critical care pediatric patients [25]. Like other authors, we believe that pain scores are central to our ability to evaluate and titrate pain relief [26].

However, the use of clinical scales has a series of disadvantages, such as the inability to perform them continuously, the degree of subjectivity, and the care burden involved for the health staff. Thus, the clinical potential of a clinical monitoring system like the NIPE. The NIPE is a
0-100 non-invasive index, calculated from heart rate variability analysis, which provides a continuous measurement of the parasympathetic tone as a surrogate for the analgesia-nociception balance, with high values corresponding to maximal parasympathetic activity (analgesia) and low values corresponding to sympathetic activation (nociception) [16]. Its use has been validated in children up to 2 years of age.

In adult patients, this monitoring system, called the Analgesia Nociception Index (ANI), has demonstrated that the respiratory variability of heart rate decreases in response to painful stimuli during general anesthesia or in post-anesthesia care unit [27-29], and it has also been used to evaluate pain in awake surgical patients [14], and in pregnant women during labor [30]. There are much less studies carried out in pediatric patients compared to adults with this monitoring system, the majority being focused on the field of anesthesia $[17,19,31]$ and very few in neonates and preterm neonates $[32,33]$.

As far as we know, the data presented here are the first regarding the usefulness of the NIPE in children with spontaneous breathing admitted to a PICU with no surgery performed. In the present study, the NIPE appears to be a sensitive tool to detect changes during nebulization, as a variation was detected in the NIPE values of 9 points during nebulization. However, these changes were almost identical between one nebulization system and the other. These results contrast with the variations in comfort that the clinical CBS detected during nebulization, as well as the greater clinical discomfort of the JN nebulization system compared to the NHF [20]. If a score of less than 17 on the CBS scale, like that determined by Van Dijk et al., is taken as a cut-off 
Table 2 Patients characteristics during nebulization

\begin{tabular}{|c|c|c|c|}
\hline Variables & $\begin{array}{l}\text { NHF } \\
N=40\end{array}$ & $\begin{array}{l}\mathrm{JN} \\
\mathrm{N}=44\end{array}$ & $P$ value \\
\hline \multicolumn{4}{|l|}{ Heart rate (beats/min) } \\
\hline Before nebulization & $126(111-141)$ & $137(123-146)$ & NS \\
\hline During nebulization & $130(114-142)$ & $141 *(126-158)$ & 0.005 \\
\hline After nebulization & $132(116-145)$ & $136(127-148)$ & NS \\
\hline \multicolumn{4}{|l|}{ Breathing frequency (bpm) } \\
\hline Before nebulization & $28(22-33)$ & $25(22-33)$ & NS \\
\hline During nebulization & $28(25-32)$ & $31 *(27-38)$ & 0.028 \\
\hline After nebulization & $28(23-33)$ & $29(23-35)$ & NS \\
\hline \multicolumn{4}{|l|}{ Oxygen saturation (\%) } \\
\hline Before nebulization & $98(97-100)$ & $99(97-100)$ & NS \\
\hline During nebulization & $99(97-100)$ & $100(97-100)$ & NS \\
\hline After nebulization & 99 (96-99) & $99(97-100)$ & NS \\
\hline \multicolumn{4}{|l|}{ Fraction of inspired oxygen } \\
\hline Before nebulization & $30(25-40)$ & $33(25-40)$ & NS \\
\hline During nebulization & $30(25-40)$ & $35(25-40)$ & NS \\
\hline After nebulization & $30(25-40)$ & $30(25-40)$ & NS \\
\hline Parent's arms & $2(5 \%)$ & $6(14 \%)$ & NS \\
\hline Time after eating & & & NS \\
\hline$>1 \mathrm{~h}$ & $29(73 \%)$ & $31(70 \%)$ & \\
\hline$\leq 1 \mathrm{~h}$ & $11(28 \%)$ & $13(30 \%)$ & \\
\hline Time of the day & & & NS \\
\hline Day time (8:00-22:00) & $29(73 \%)$ & $37(84 \%)$ & \\
\hline Night time (22:00-8:00) & $11(28 \%)$ & $7(16 \%)$ & \\
\hline Use of analgesics & 0 & $1(2 \%)$ & NS \\
\hline
\end{tabular}

Quantitative variables are expressed as median (interquartile range). Categorical values are expressed as $\mathrm{n}(\%)$

$N H F$ nebulization system integrated in High-Flow nasal cannula, $J N$ jet nebulizer

$* \mathrm{P}<0.05$ obtained by Wilcoxon test comparing before and during nebulization. $\mathrm{P}$ value for each variable between NHF and JN. P value $<0.05$ was considered statistically significant

point for the administration of analgesics [34], the number of patients with values below 17 during nebulization was significantly higher with JN than with NHF.

This lack of correlation between the comfort difference during the nebulization with the NIPE and the measurement with the clinical scales has also been found in adult patients $[14,35]$. Furthermore, there could be other explanations for these findings in our study. In the first place, an increase of 4 points was found in the HR compared to the baseline, there being a slight correlation with the change in the clinical CBS. This increase of barely $1 \%$ difference from the increase found in other studies supports changes in the HR of $10-25 \%$ as a good indicator of nociception $[17,36]$. In pediatrics, and in pediatric anesthetics in particular, it is normal practice to monitor changes in HR from baseline as an indicator of pain and discomfort [37, 38]. As such, Migeon et al. showed that ANI measurements decreased after skin incision in children over 2 years of age anesthetized with sevoflurane. This could be used in combination, or as an alternative to HR monitoring [17]. Ledowski et al. observed similar results, concluding that ANI might provide a more sensitive assessment of nociception in anesthetized children than hemodynamic parameters or skin conductance [39]. However, the percentage rise in HR required to identify a painful stimulus in children is debatable, some studies not finding changes in the HR in painful procedures [40, 41], or even emphasizing the need for caution in interpreting heart rate as an index of comfort [42]. Secondly, we had to exclude a number of NIPE values with Quality ' 0 ' which meant not analyzing $18 \%$ of the nebulizations. However, the number of nebulizations rejected in each nebulization system was similar; thus this should not be the reason why we did not find any differences between the two nebulizers used.

In any case, it is worth noting, given that the NIPE system depends on the quality of the ECG signal, that it may have limitations in awake infants whose continuous movement interferes in the ECG trace. This fact could explain the contrast with the potential of this system in older pediatric patients. Nonetheless, these are the children in whom pain assessment can be the most challenging.

Third, a limitation of our study is that, owing to its design, it does not permit determination of the NIPE values at the exact same time as the clinical scales. The non-integration of the monitor as a routine tool in our unit meant that the exact time of the evaluation of the scale will not be noted correctly in the monitor, obliging us to rule out this line of study, and focus instead on analysis of the difference of the values obtained during the entire period. In future research we aim to measure not only the magnitude of NIPE variation during nebulization, as was done in this present paper, but to investigate whether the direction of this change (increase or decrease) indeed correlates to the variations in comfort levels measured using clinical scales.

Finally, we cannot rule out the possibility that factors such as noise, and other environmental factors common to a PICU, might have influenced the values recorded by the NIPE [42, 43]. Although randomization of the nebulization of each system would have decreased this bias, these factors were not analyzed.

In summary, this study demonstrates the variation in discomfort during nebulization in the patient with bronchiolitis with NIPE monitoring, although it does not seem to correlate well with the clinical evaluation. Clinical scales like the Comfort Behavior Scale are good tools to evaluate the discomfort and pain generated by nebulization in nonsedated patients breathing spontaneously, presenting with bronchiolitis. More studies are needed before being able to recommend the NIPE monitor as an alternative to the evaluation of comfort by more traditional methods, such as HR monitoring and the use of clinical scales. 
Acknowledgements We are grateful to MMDOLORIS for providing a NIPE monitor for this study.

\section{Compliance with ethical standards}

Conflict of interest No conflicts of interest declared.

\section{References}

1. Viswanathan M, King VJ, Bordley C, Honeycutt AA, Wittenborn J, Jackman AM, Sutton SF, Lohr KN. Management of bronchiolitis in infants and children. Evid Rep Technol Assess (Summ). 2003;69:1-5.

2. American Academy of Pediatrics, Subcommittee on Diagnosis and Management of Bronchiolitis. Diagnosis and management of bronchiolitis. Pediatrics. 2006;118(4):1774-93.

3. Meissner HC. Viral bronchiolitis in children. N Engl J Med. 2016;374(1):62-72.

4. Deshpande SA, Northern V. The clinical and health economic burden of respiratory syncytial virus disease among children under 2 years of age in a defined geographical area. Arch Dis Child. 2003;88(12):1065-9.

5. Ralston SL, Lieberthal AS, Meissner HC, Alverson BK, Baley JE, Gadomski AM, Johnson DW, Light MJ, Maraqa NF, Mendonca EA, Phelan KJ, Zorc JJ, Stanko-Lopp D, Brown MA, Nathanson I, Rosenblum E, Sayles S 3rd, Hernandez-Cancio S, American Academy of Pediatrics. Clinical practice guideline: the diagnosis, management, and prevention of bronchiolitis. Pediatrics. 2014;134(5):e1474-502.

6. Atigapramoj NS. Bronchiolitis: what's all the fuss about? J Emerg Med. 2018;54(1):127-8.

7. Walsh P, Rothenberg S. American Academy of Pediatrics 2014 bronchiolitis guidelines: bonfire of the evidence. West J Emerg Med. 2015;16(1):85-8.

8. Castro-Rodriguez JA, Rodriguez-Martinez CE, Sossa-Briceño MP. Principal findings of systematic reviews for the management of acute bronchiolitis in children. Paediatr Respir Rev. 2015 Sep;16(4):267-75.

9. Carsin A, Sauvaget E, Bresson V, Retornaz K, Cabrera M, Jouve E, Truillet R, Bosdure E, Dubus JC. Early halt of a randomized controlled study with $3 \%$ hypertonic saline in acute bronchiolitis. Respiration. 2017;94(3):251-7.

10. Harris J, Ramelet AS, van Dijk M, Pokorna P, Wielenga J, Tume L, Tibboel D, Ista E. Clinical recommendations for pain, sedation, withdrawal and delirium assessment in critically ill infants and children: an ESPNIC position statement for healthcare professionals. Intensive Care Med. 2016;42(6):972-86.

11. Malviya S, Voepel-Lewis T, Tait AR, Merkel S, Lauer A, Munro $\mathrm{H}$, Farley F. Pain management in children with and without cognitive impairment following spine fusion surgery. Paediatr Anaesth. 2001;11(4):453-8.

12. Brand K, Thorpe B. Pain assessment in children. Anaesth Intensive Care Med. 2016;17:270-3.

13. Morton NS. Pain assessment in children. Paediatr Anaesth. 1997;7(4):267-72.

14. Ledowski T, Tiong WS, Lee C, Wong B, Fiori T, Parker N. Analgesia nociception index: evaluation as a new parameter for acute postoperative pain. Br J Anaesth. 2013;111(4):627-9.

15. Jeanne M, Logier R, De Jonckheere J, Tavernier B. Heart rate variability during total intravenous anesthesia: effects of nociception and analgesia. Auton Neurosci Basic Clin. 2009;147(1-2):91-6.

16. Jeanne M, Logier R, De Jonckheere J, Tavernier B. Validation of a graphic measurement of heart rate variability to assess analgesia/nociception balance during general anesthesia. Conf Proc IEEE Eng Med Biol Soc. 2009;2009:1840-3.

17. Migeon A, Desgranges FP, Chassard D, Blaise BJ, De Queiroz M, Stewart A, Cejka JC, Combet S, Rhondali O. Pupillary reflex dilatation and analgesia nociception index monitoring to assess the effectiveness of regional anesthesia in children anesthetised with sevoflurane. Paediatr Anaesth. 2013;23(12):1160-5.

18. Avez-Couturier J, De Jonckheere J, Jeanne M, Vallée L, Cuisset J-M, Logier R. Assessment of procedural pain in children using analgesia nociception index: a pilot study. Clin J Pain. 2016;32(12):1100-4.

19. Carnavale FA, Razack S. An item analysis of the COMFORT scale in a pediatric intensive care unit. Pediatr Crit Care Med. 2002;3(2):177-80. 4p.

20. Valencia-Ramos J, Mirás A, Cilla A, Ochoa C, Arnaez J. Incorporating a nebulizer system into high flow nasal cannula improves comfort in infants with bronchiolitis. Respir Care. 2018;63:886-93.

21. McGrath PJ, Frager G. Psychological barriers to optimal pain management in infants and children. Clin J Pain. 1996;12(2):135-41.

22. Ghazaly M, Nadel S. Characteristics of children admitted to intensive care with acute bronchiolitis. Eur J Pediatr. 2018;177(6):913-20.

23. Kou M, Hwang V, Ramkellawan N. Bronchiolitis. From practice guideline to clinical practice emerg. Med Clin North Am. 2018;36(2):275-86.

24. Van Dijk M, De Boer JB, Koot HM, Tibboel D, Passchier J, Duivenvoorden HJ. T The reliability and validity of the COMFORT scale as a postoperative pain instrument in 0 to 3 -year-old infants. Pain. 2000;84(2-3):367-77.

25. Bosch-Alcaraz A, Falcó-Pegueroles A, Jordan I. A literature review of comfort in the paediatric critical care patient. J Clin Nurs. 2018;27:2546-57.

26. Valkenburg V, Boerlage AJ, Ista AA, Duivenvoorden E, Tibboel HJ, D, Dijk M Van. The COMFORT-Behavior scale is useful to assess pain and distress in 0- to 3-year-old children with Down syndrome. Pain. 2011;152(9):2059-64.

27. Jeanne J, Clément M, De Jonckheere C, Logier J, Tavernier R. $\mathrm{B}$. Variations of the analgesia nociception index during general anaesthesia for laparoscopic abdominal surgery. J Clin Monit Comput. 2012;26(4):289-94.

28. Boselli E, Bouvet L, Bégou G, et al. Prediction of immediate postoperative pain using the analgesia/nociception index: a prospective observational study. Br J Anaesth. 2014;112(4):715-21.

29. Boselli E, Logier R, Bouvet L, Allaouchiche B. Prediction of hemodynamic reactivity using dynamic variations of Analgesia/Nociception Index ( $\triangle \mathrm{ANI})$. J Clin Monit Comput. 2016;30(6):977-84.

30. Le Guen M, Jeanne M, Sievert K, Al Moubarik M, Chazot T, Laloë PA, Dreyfus JF, Fischler M. The Analgesia Nociception Index: a pilot study to evaluation of a new pain parameter during labor. Int J Obstet Anesth. 2012;21(2):146-51.

31. Gall O, Champigneulle B, Schweitzer B, Deram T, Maupain O, Montmayeur Verchere J, Orliaguet G. Postoperative pain assessment in children: a pilot study of the usefulness of the analgesia nociception index. Br J Anaesth. 2015;115(6):890-5.

32. Cremillieux C, Makhlouf A, Pichot V, Trombert B, Patural H. Objective assessment of induced acute pain in neonatology with the Newborn Infant Parasympathetic Evaluation index. Eur J Pain. 2018;22:1071-9.

33. Rakza T, Butruille L, Thirel L, Houfflin-Debarge V, Logier R, Storme L, De Jonckheere J. Short-term impact of assisted deliveries: evaluation based on behavioral pain scoring and heart rate variability. Clin J Pain. 2018;34(5):445-9. 
34. van Dijk M, Peters JWB, van Deventer P, Tibboel D. The COMFORT Behavior Scale: a tool for assessing pain and sedation in infants. Am J Nurs. 2005;105(1):33-6.

35. Szental JA, Webb A, Weeraratne C, Campbell A, Sivakumar H, Leong S. Postoperative pain after laparoscopic cholecystectomy is not reduced by intraoperative analgesia guided by analgesia nociception index (ANI®) monitoring: a randomized clinical trial. Br J Anaesth. 2015;114(4):640-5.

36. Oberndorfer U, Marhofer P, Bösenberg A, Willschke H, Felfernig M, Weintraud M, Kapral S, Kettner SC. Ultrasonographic guidance for sciatic and femoral nerve blocks in children. Br J Anaesth. 2007;98(6):797-801.

37. Ecoffey C, Lacroix F, Giaufré E, Orliaguet G, Courrèges P, Association des Anesthésistes Réanimateurs Pédiatriques d'Expression Française (ADARPEF). Epidemiology and morbidity of regional anesthesia in children: a follow-up one-year prospective survey of the French-Language Society of Paediatric Anaesthesiologists (ADARPEF). Paediatr Anaesth. 2010;20(12):1061-9.

38. Naja Z, Al-Tannir MA, Faysal W, Daoud N, Ziade F, El-Rajab M. A comparison of pudendal block vs dorsal penile nerve block for circumcision in children: a randomised controlled trial. Anaesthesia. 2011;66(9):802-7.

39. Ledowski T, Averhoff L, Tiong WS, Lee C. Analgesia Nociception Index (ANI) to predict intraoperative haemodynamic changes: results of a pilot investigation. Acta Anaesthesiol Scand. 2014;58(1):74-9.

40. Lamas A, López-Herce J, Sancho L, Mencía S, Carrillo A, José Santiago M, Martínez V. Responsiveness to stimuli of bispectral index, middle latency auditory evoked potentials and clinical scales in critically ill children. Anaesthesia. 2008;63(12):1296-301.

41. Cury MRJ, Martinez FE, Carlotti APCP. Pain assessment in neonates and infants in the post-operative period following cardiac surgery. Postgrad Med J. 2013;89(1048):63-7.

42. Foster RL, Yucha CB, Zuk J, Vojir CP. Physiologic correlates of comfort in healthy children. Pain Manag Nurs. 2003;4(1):23-30.

43. Carnevale FA, Gaudreault J. The experience of critically ill children: a phenomenological study of discomfort and comfort. Dynamics. 2013;24(1):19-27.

Publisher's Note Springer Nature remains neutral with regard to jurisdictional claims in published maps and institutional affiliations. 